\title{
Case Report and Review of Literature on Anaesthetic Management of Ruptured Splenic Pregnancy with Hypovolemic Shock.
}

\author{
${ }^{1}$ Sugam Preet Kaur, ${ }^{2}$ Deepika Sareen, ${ }^{3}$ Vishwas Sathe, ${ }^{4}$ Olvyna D'souza, \\ ${ }^{5}$ Hemesh Shewale \\ ${ }^{1}$ junior Resident Year Department of Anaesthesia Mgm Medical College \& Hospital \\ Kamothe, Navi Mumbai, Maharashtra, India \\ ${ }^{2}$ assistant Professor Department of Anaesthesia Mgm Medical College \& Hospital Kamothe, Navi Mumbai, \\ Maharashtra, India \\ ${ }^{3}$ department of Anaesthesia Mgm Medical College \& Hospital Kamothe, Navi Mumbai, Maharashtra, India \\ ${ }^{4}$ department of Anaesthesia Mgm Medical College \& Hospital Kamothe, Navi Mumbai-410209, Maharashtra, \\ India \\ ${ }_{5}^{5}$ junior Resident Year Department of Anaesthesia Mgm Medical College \& Hospital Kamothe, Navi Mumba \\ Maharashtra, India
}

\begin{abstract}
Splenic pregnancy is the least common form of extrauterine pregnancy. Recognition of this rare form of gestation is of critical importance, owing to the risk of exsanguination and death. We report a case of 19 year old female with ruptured splenic pregnancy resulting in hypovolemic shock. Emergency exploratory laparotomy was performed and was successfully managed under general anaesthesia.
\end{abstract}

Keywords: Ectopic splenic pregnancy, Hypovolemic shock,Early detection, Rapid Sequence induction(RSI).

\section{Introduction}

Ectopic pregnancy occurs when a fertilized ovum implants somewhere other than the uterine cavity and it occurs with an estimated incidence of 19.7 per 1000 pregnancies $^{1}$. The common site of ectopic implantation is within fallopian tube, which is accounting for $95.5 \%$ of all ectopic gestation ${ }^{2}$. Approximately $1.3 \%$ of ectopic pregnancies are abdominal and occur with direct implantation onto the peritoneal surface ${ }^{2}$. The splenic pregnancy is one of the rarest sites for ectopic pregnancy ${ }^{3}{ }^{4}$. Because of abundant blood supply, splenic ectopic pregnancy is prone to cause massive peritoneal bleeding and patient may present with hypovolemic shock ${ }^{5}$. Early recognition and early diagnosis of ectopic pregnancy is critical for saving patient's life.

\section{Case Report}

A 19 year old female, American Society of Anaesthesiologists physical status II (E), primigravida, not registered for antenatal checkup elsewhere, presented to the Emergency department with history of amenorrhea since 8 weeks with severe abdominal pain and vomiting. She denied history of smoking and alcohol. Urine pregnancy test and $\beta$-hCG test was positive. USG abdomen revealed normal ovaries, no gestational sac seen in uterus with moderate to severe fluid collection seen in abdomen and pelvis. A provisional diagnosis of acute abdomen was made and patient was scheduled for an emergency exploratory laparotomy for suspected ruptured ectopic pregnancy with hemoperitoneum.

On preanaesthetic evaluation, patient was in obvious distress. She was 164 inches tall and weighed 60 $\mathrm{kg}$ with pulse rate of $122 / \mathrm{min}$, regular in rhythm, of low volume and Blood pressure of $82 / 60 \mathrm{~mm} \mathrm{Hg}$ taken on right upper arm in supine position with severe pallor. On airway assessment the patient had adequate mouth opening, no loose teeth, caps or crowns and with Mallampati criteria II. Preoperative laboratory workup revealed Hemoglobin of $7.5 \mathrm{gm} / \mathrm{dl}$. Platelet count, total blood count, prothrombin time, international normalized ratio were normal. Serum creatinine was $1.3 \mathrm{mg} / \mathrm{dl}$. Blood products including 4 units of packed cells and 4 units of fresh frozen plasma were ordered.

General anaesthesia was planned for the exploratory laparotomy as the patient was in a state of shock and was hypotensive. Informed consent was obtained after the risk of extensive bleeding, possible blood transfusion, general anaesthesia related complications were explained to the patient .Two $18 \mathrm{G}$ intravenous line inserted and 1.5 litres of crystalloid administered preoperatively. In the operation room, standard ASA monitors were applied. The patient was pre medicated with Inj Ranitidine $50 \mathrm{mg}$. Inj Metoclopromide $10 \mathrm{mg}$, Inj Glycopyrolate $0.2 \mathrm{mg}$ and Inj Fentanyl $50 \mu \mathrm{g}$. Rapid sequence intubation was carried out with INJ Ketamine 60 $\mathrm{mg}$ and Inj Succinylcholine $100 \mathrm{mg}$ with cuffed endotracheal tube of $7.5 \mathrm{~F}$ and fixed at mark $21 \mathrm{~cm}$ after 
confirmation of bilateral air entry. Anaesthesia was maintained with titrated doses of ISOFLURANE and intermittent boluses of Inj Fentanyl. Inj Atracurium was used for neuromuscular blockade.

On opening the abdomen with a midline vertical incision, chorionic decidual type tissue was noted on superior pole of spleen with active bleeding present from splenic pole. No other bleeding site was noted. Splenorrhaphy was done after the removal of gestational sac. Extensive haemorrhage was noted. Estimated blood loss during the surgery was $2500 \mathrm{ml}$. Intra-operatively; patient's blood pressure was managed with crystalloids and 6\% hydroxyl-ethyl starch along with 12-15 mg of Inj Ephedrine. Patient received 2 units of fresh frozen plasma followed by 3 units of packed cell volume intra-operatively and 1 unit of packed cell volume postoperatively. Hypothermia was prevented using intra-venous fluid warmer and active convective warming of the patient. Blood gas analysis revealed metabolic acidosis with $\mathrm{pH}$ value of 7.O, $160 \mathrm{ml}(12 \mathrm{gm})$ of sodium bicarbonate was infused and hypocalcaemia was corrected with $1 \mathrm{gm}$ of intravenous calcium gluconate. Total duration of surgery was 280 minutes which was uneventful post the fluid and blood resuscitation. The emergence, extubation and recovery were uneventful. On extubation, patient was hemodynamically stable with heart rate of $88 / \mathrm{min}$, blood pressure of $116 / 54 \mathrm{~mm} \mathrm{Hg}$ taken on right arm in supine position, respiratory rate of $14 /$ minute and $\mathrm{SPO} 2$ of $99 \%$ on $35 \% \mathrm{FIO} 2$.

Her postoperative period was uneventful and she was discharged 4 days after surgery. Specimen pathology confirmed splenic pregnancy.

\section{Discussion}

Abdominal pregnancies are classified as either primary or secondary; the latter, which are much more common, are associated with displacement of the fertilized ovum from the fallopian tubes or the uterus to a secondary site. ${ }^{4} 8$ Primary abdominal pregnancies, which arise from fertilization of an ovum within the peritoneal cavity, with anatomically normal fallopian tubes, ovaries, and uterus, are extremely rare. ${ }^{4}$ According to Studdiford ${ }^{8}$, the criteria for primary abdominal pregnancy are as follows: (1) fallopian tubes and ovaries are grossly normal and show no evidence of recent injury; (2) no evidence of uteroplacental fistula; and (3) a pregnancy of no more than 12 weeks' gestation with trophoblastic elements related exclusively to a peritoneal surface. The third criterion ensures that the pregnancy is immature enough to exclude the possibility of secondary implantation to the peritoneal cavity after primary tubal pregnancy rupture. In our case, during intraoperative examination, fallopian tubes were normal, no other abnormalities were identified. There was no evidence of pregnancy outside the spleen, therefore satisfying the criteria established for primary splenic pregnancy

Our review of previously published reports of primary splenic pregnancies (see Table) revealed a mean age of 27.3 years (range, 23-37 years). As with our case, most patients presented with sudden-onset left upper quadrant abdominal pain that radiated to the left shoulder. Eight of the 9 previously published cases had preoperative diagnoses of ruptured ectopic pregnancy, with the remaining case undiagnosed until the results of histological examination. All patients survived. A variety of splenic implantation sites have been reported and range from superior to lower pole and hilum. Most gestations manifested as capsular projections and all were sub capsular in location.

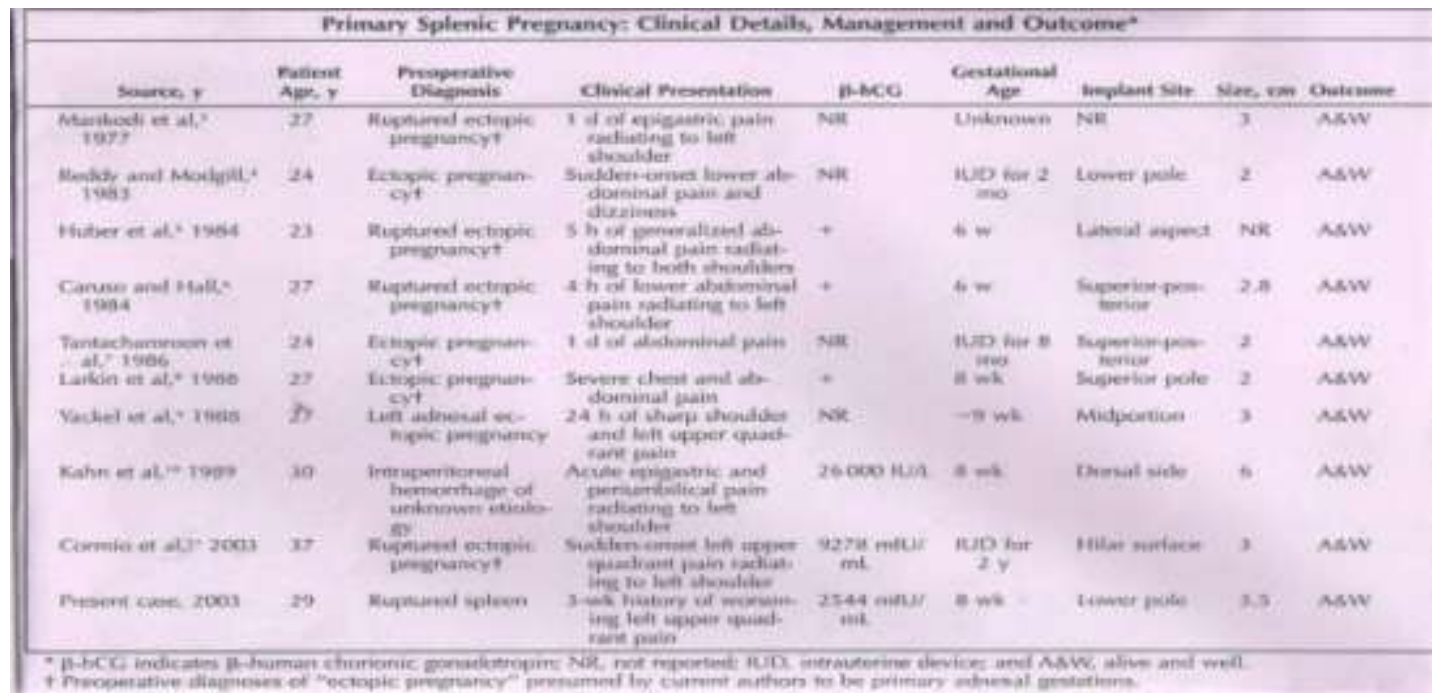

The spleen is relatively more favorable for implantation considering the fact that it is a flat organ, rich in blood flow, and easily reached in the human supine position by the fertilized ovum. However, none of the 
anatomic sites described above, including the spleen, can accommodate placental attachment or a growing embryo; therefore, rupture and a massive hemorrhage may very likely occur if left untreated. ${ }^{7}$

Several factors are known to increase the risk of an ectopic pregnancy, including a history of pelvic inflammatory disease, a previous ectopic pregnancy, endometriosis, previous pelvic surgery, reproductive assistance, and uterotubal anomalies. ${ }^{4}$

The clinical hallmark of an ectopic pregnancy is abdominal pain and amenorrhea with vaginal bleeding, often occurring 6 to 8 weeks after the last normal menstrual period. ${ }^{7}$ Nearly all previously reported cases of splenic pregnancies presented with abdominal tenderness or intra-abdominal bleeding. ${ }^{4}$ Our patient also presented with complaints of abdominal tenderness accompanied with vomiting.

The absence of an intrauterine gestational sac on abdominal sonography in conjunction with a $\beta$-hCG level of greater than $1500 \mathrm{U} / \mathrm{L}$ is thought to be suggestive of an ectopic pregnancy ${ }^{7}$. The high blood level of the $\beta$-hCG titer was the only suggestive indicator of pregnancy, most probably an unidentified ectopic pregnancy. Experience has shown that in more than 50\% of cases, the diagnosis of an abdominal pregnancy is overlooked. Because sonography is used to confirm the presence of an intrauterine pregnancy, it is probably the most important tool in the diagnosis of an extra uterine pregnancy. ${ }^{1}$ In the case described, even though $\beta$-hCG titers were well above the discriminatory zone, sonography did not reveal an intrauterine gestational sac.

Early recognition and early diagnosis of ectopic pregnancy is important for saving patient's life. In view of the deteriorating condition of patient, decision for emergency exploratory laparotomy was taken. Considering the hypovolemic shock we decided to operate patient under general anesthesia and the drugs and techniques used to anaesthetize the patient were optimally safe. Hypovolemia secondary to bleeding from ruptured splenic pregnancy was treated by crystalloids, colloids and blood to preserve perfusion of vital organs.

\section{Conclusion}

Although recognition of primary abdominal pregnancy poses a difficult diagnostic challenge, increased detection of ectopic pregnancies is made possible through improved access to ultrasonography and improved sensitivity of urine and serum $\beta$-human chorionic gonadotropin tests. Recognition of this rare form of gestation is of critical importance owing to the risk of exsanguinations and death, and should be adequately managed. As the patient was hemodynamically unstable at presentation and required massive transfusion, the choice of anesthesia should be general anaesthesia.

\section{References}

[1]. Center for Disease Control and Prevention. Ectopic pregnancy: United States, 1990-1992. JAMA. 1995; $273: 533$

[2]. Bouyer J, Coste J, Fernandez H, Pouly JL, Job-Spira N. Sites of ectopic pregnancy: a 10-year population-based study of 1800 cases. Hum Reprod. 2002; 17: 3224-3230

[3]. Guo Gang Yang Yudong, Guoan Zhang (2010) Successful Laparoscopic Management of Early Splenic Pregnancy: Case Report and Review of Literature. The Journal of Minimally Invasive Gynecology 17:794-797.

[4]. Alexandra N Kalof, Bradbury Fuller, Maureen Harmon (2004) Splenic Pregnancy. Arch Pathol Lab Med 128: 146-148.

[5]. Youxin Ji, Ketao Lan, Keke Nie, Shichao Lliu,(2015) 'A Live splenic ectopic pregnancy '. Journal of cell science and therapy. 2015; volume 6 issue 21000201

[6]. Atrash HK, Friede A, Hogue C (1987) Abdominal pregnancy in the United States: frequency and maternal mortality. ObstetGynecol 69:333-337.

[7]. Tenore JL. Ectopic pregnancy. Am Fam Physician 2000; 61: 1080-1088

[8]. Sapuri M, Klufio C. A case of advanced viable extrauterine pregnancy. P N G Med J 1997; 40:44-47.

[9]. Studdiford, W. E. Primary peritoneal pregnancy. Am J Obstet Gynecol 1942. 44:487-491.

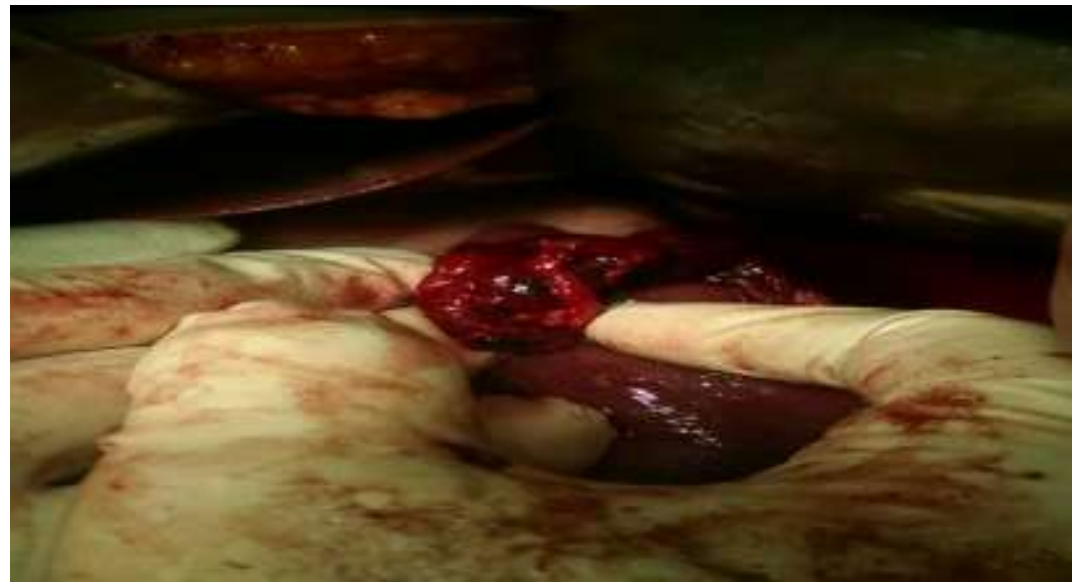

